Article

\title{
A Hybrid Compensation Topology for Battery Charging System Based on IPT Technology
}

\author{
Junfeng Yang ${ }^{1}$, Xiaodong Zhang ${ }^{1, *}$, Xu Yang ${ }^{1}$, Qiujiang Liu ${ }^{1}$ and Yi Sun ${ }^{2}$ \\ 1 School of Electrical Engineering, Beiijing Jiaotong University, Beijing 100044, China; \\ yangjunfeng@bjtu.edu.cn (J.Y.); 17117425@bjtu.edu.cn (X.Y.); 14117383@bjtu.edu.cn (Q.L.) \\ 2 China Electrotechnical Society, Beijing 100055, China; sunyi@ces.org.cn \\ * Correspondence: xdzhang@bjtu.edu.cn; Tel.: +86-51687108
}

Received: 7 September 2019; Accepted: 2 October 2019; Published: 9 October 2019

\begin{abstract}
Based on the double-sided LCC (DLCC) compensation topology circuit, a battery charging method is proposed to meet various charging requirements. Firstly, mathematical model was obtained by modeling primary and secondary sides of DLCC. The current gain and voltage gain of the inductive power transfer (IPT) system are derived. Then, taking into account the smooth conversion of charging mode, the parameter configuration conditions for constant current (CC) output and constant voltage (CV) output are designed systematically. Finally, after choosing parameters, the CC and CV modes can be achieved by adding one switch and an auxiliary capacitor. With few additional components and non-sophisticated control, both cost and complexity can be significantly reduced. An experimental prototype with $64 \mathrm{~V}$ charging voltage and $1 \mathrm{~A}$ charging current is built. The experimental results show that the charging voltage and current fluctuation of the system are small and the method can meet the above requirements.
\end{abstract}

Keywords: inductive power transfer (IPT); LCC compensation network; constant current (CC); constant voltage $(\mathrm{CV})$

\section{Introduction}

With the advantages of convenience, safety, and being environment-friendly, inductive power transfer (IPT) has attracted considerable attention in low-power biomedical implants [1], medium-power consumer electronics [2], high-power electric vehicles (EVs) [3-5], and so on [6-8]. The promising technology utilizes a time-varying electromagnetic field to transfer energy without cable. Recently, high performance lithium-ion batteries have been widely used in various energy storage applications due to their high energy density, self-discharge rate, and negligible memory effect $[9,10]$. The charging method of batteries is one of the key technologies to realize popularization. A typical charging profile of a battery comprises constant output current (CC) charging mode and constant output voltage (CV) charging mode $[5,11]$. To be specific, initially, the battery is charged in CC mode which means the output current is constant, and the battery voltage increases rapidly. When the battery voltage reaches a specified voltage, the charger changes to the $\mathrm{CV}$ mode with the constant output voltage until the battery current reaches nearly zero. The equivalent resistance of the battery varies significantly during the entire charging profile, which makes the design of the IPT charger complicated. To prolong the battery lifetime and recycle time, an IPT charger should provide expected output current and voltage efficiently for different charging stages of the battery.

In order to satisfy the battery charging curve (from CC mode to CV mode), lots of approaches for IPT system have been proposed. These approaches can be roughly divided into two categories: control schemes and hybrid compensation topologies. According to the different control objects, control methods can be classified into primary side control and secondary side control. For secondary side 
control, the regulation of output current and voltage depends on an extra DC-DC converter [12]. This approach will lead to the change of the secondary side reflection impedance, which makes the energy picked up by the magnetic coupler vary greatly and its output stabilization ability is limited. A front-end converter added before the primary-side inverters is also applied in [13], however, this approach requires additional components and increases the associated energy losses. Besides, for continued regulation, a wireless communication between the primary side and the secondary side is needed. The high-frequency inverter (HFI) is another critical and indispensable converter to regulate the output voltage of the source. Load-independent output current or output voltage can be realized by phase-shift control (PSC) [14] or variable frequency control (VFC) [9,15-17]. However, it is difficult to achieve zero-voltage switching under light load condition with PSC and the frequency bifurcation phenomenon which would lose controllability and stability, which may occur under variable load with VFC. Obviously, the additional power converter makes the whole system not only inefficient, bulky, and uneconomical, but also more complicated.

Besides the sophisticated control strategies which have been aforementioned, some researchers have focused their research on the new topologies to realize load-independent output current and voltage without control strategies. There are four basic compensation topologies: series-series (SS), series-parallel (SP), parallel-series (PS), and parallel-parallel (PP). Based on the four topologies, some dual hybrid topologies to realize CC and CV modes are put forward [13], [18-20]. In [18], it is realized by changing topology between SS and SP with different parameters, but the circuit is complicated, containing a center-tapped loosed transformer, four switches, and two additional capacitors. In [19], an additional capacitor and two switching at the primary side form a hybrid topology of SS-PS. In [20], after full discussion of series connected and parallel connected compensation circuits, two hybrid topologies are proposed by switching between either SP and PP topology or SS and PS topology. Nevertheless, not only are three switches and an additional inductance introduced, but the wireless link is also required.

Another hybrid topology combining high-order compensation topologies with seven additional components is proposed in [21-23]. In [21], an auxiliary capacitor and two switches are employed at the receiver side, which the basic topology adopts S-LCC with a series (S) compensation network on the primary side and an inductor-capacitor-capacitor (LCC) compensation network on the secondary. In [22], CC and CV outputs are realized by switching between double-sided LCC (DLCC) and LCC-S modes. The output of HFI can achieve zero phase angle (ZPA) when using two switches and an auxiliary capacitor. Based on double-sided inductor-capacitor-inductor (DLCL) compensation alteration, CC mode and CV mode are achieved in [23]. The compensation tank consists of one additional switch, one auxiliary capacitor, and two auxiliary inductors. What is more, the process of switching is not analyzed without considering continuous charging under two modes.

Because of the lack of freedom, the four basic compensations may not be able to meet the required output, which is difficult to optimize design. Thus, higher order compensation topologies are better to apply to implement CC and CV output. Although some have been used in aforementioned work, each has its own shortcomings. Since the LCC topology has three parameters design freedoms [24-26], it will be better to implement this by changing the components of the topology. This paper develops a new IPT charging method to meet various charging requirements, including five sections. In Section 2, simplified circuits of DLCC topology and the output characteristics are theoretically analyzed. Detailed systematic analysis of parameters to achieve constant voltage and current output is also presented. Considering the smooth transition between two modes, the parametric design method of LCC network is carried out in Section 3. In Section 4, an experimental prototype is implemented and experimental results are presented in detail to evaluate the performance of the designed IPT system. The experimental results are in good agreement with the performance of the method, indicating that the method proposed in the paper is effective and feasible. 


\section{Analysis of Circuits}

\subsection{System Topology}

As Figure 1 shows, the typical IPT charging system consists of three parts; power supply, resonant tank, and load. The power supply consists of a constant voltage source and a high-frequency full-bridge square-wave inverter. The resonant tank includes primary and secondary compensation networks, and magnetic coupled coils. The load includes a full bridge rectifier, a filter, and a battery load.

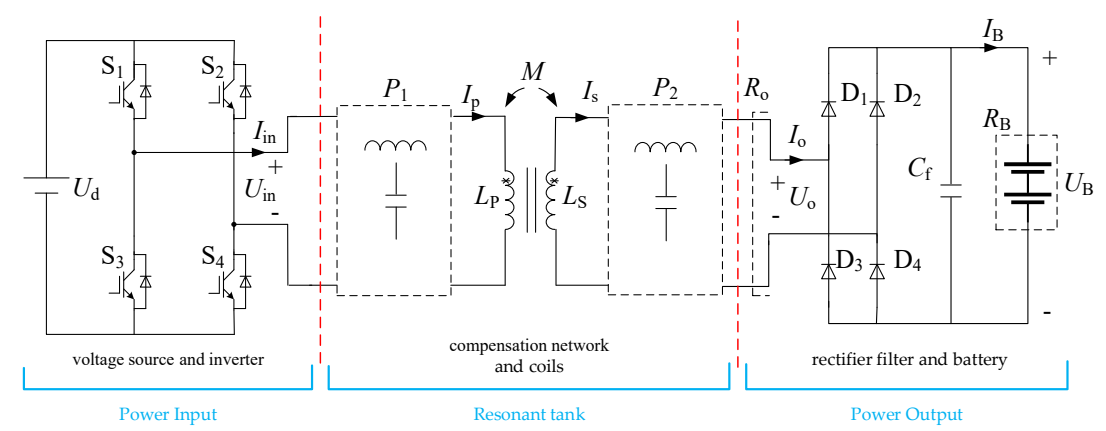

Figure 1. Wireless battery charging system.

In this paper, the resonant tank is what we have focussed on. The input voltage of the resonator is the square wave obtained by the high frequency inverter (HFI). Owing to the filtering function of compensation network, the current of primary coil is nearly sinusoidal, containing only the fundamental component. As a result, the fundamental harmonic approximation (FHA) is employed. When the duty cycle of the inverter is 50\%, the relationship between the inverters' AC output RMS voltage $U_{\text {in }}$ and the input DC voltage $U_{d}$ can be expressed in the equation as follows:

$$
U_{i n}=\frac{2 \sqrt{2}}{\pi} U_{d}
$$

The relationship between input voltage $U_{O}$, current $I_{O}$ of full bridge rectifier, and charging voltage $U_{B}, I_{B}$ at the power receiver can be expressed as follows:

$$
\left\{\begin{array}{c}
U_{O}=\frac{2 \sqrt{2}}{\pi} U_{B} \\
I_{O}=\frac{\sqrt{2} \pi}{4} I_{B}
\end{array}\right.
$$

The equivalent resistance of the battery load to the resonant tank is:

$$
R_{O}=\frac{8}{\pi^{2}} R_{B}
$$

To facilitate the analysis of the inductive power transfer characteristics, the fundamental wave equivalent only containing the compensation topology and the coil system was considered. $U_{\text {in }}, I_{\text {in }}$, $U_{O}, I_{O}, R_{O}$ derived from Figure 1 were adopted to analyze at the primary side and the secondary side.

\subsection{Realization of CC Mode and CV Mode}

Varied from T-type network, the inductor-capacitor-inductor (LCL) compensation network is promoted widely for its good output characteristics. By adding another capacitance to the coil branch, which decreases the device pressure of compensate inductance and improves the power transmission capability, the LCC compensation network was built. According to the T-type compensation, the equivalent circuit of double-side LCC is put forward in Figure 2. $M$ is the mutual inductance of transmitting and receiving coils, and the parasitic resistance of the capacitance and inductance coil is ignored. $U_{i n}$ is the AC input voltage source after HFI. $L_{P}$ and $L_{S}$ represent the self-inductance of 
the transmitting and receiving coils, respectively. $L_{1}, C_{1}$, and $C_{2}$ form the primary side LCC resonant compensation network, and $L_{4}, C_{3}$, and $C_{4}$ form the secondary side LCC resonant compensation network. In order to facilitate the analysis, $L_{2}$ was used instead of the branch composed of $C_{2}$ and $L_{P}$, and $L_{3}$ was used instead of the branch composed of $C_{3}$ and $L_{S}$.

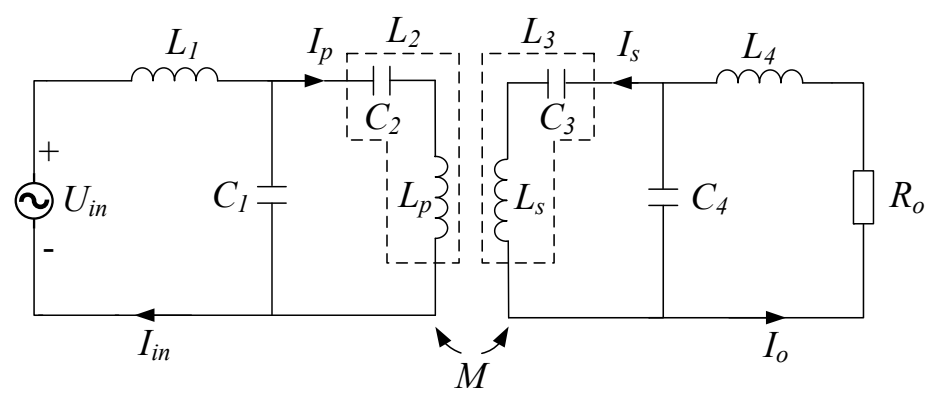

Figure 2. Circuit of DLCC compensation topology.

Firstly, the primary side was analyzed. Assuming that the secondary impedance is $Z_{s}$ and the primary side impedance reflected from the secondary side is $Z_{r}$, the overall input impedance of HFI is

$$
\begin{aligned}
Z_{i n} & =j \omega L_{1}+\left(\frac{1}{j \omega C_{1}}\right)\left(\frac{1}{j \omega C_{2}}+j \omega L_{p}+Z_{r}\right) \\
& =\frac{Z_{r}+j \omega\left(L_{2} A_{1}-C_{2} Z_{r}^{2}+L_{1} A_{1}^{2}+L_{1} A_{2}^{2}\right)}{A_{1}^{2}+A_{2}^{2}}
\end{aligned}
$$

where

$$
Z_{r}=\frac{(\omega M)^{2}}{Z_{s}} A_{1}=1-\omega^{2} C_{1} L_{2} A_{2}=\omega C_{1} Z_{r} L_{2}=L_{P}-\frac{1}{\omega^{2} C_{2}}
$$

The $w$ is the angular frequency. The current flowing through the transmitting coil can be expressed as

$$
I_{p}=\frac{U_{i n}}{\left(1-\omega^{2} C_{1} L_{1}\right) Z_{r}-j \omega\left(\omega^{2} C_{1} L_{2} L_{1}-L_{1}-L_{2}\right)}
$$

The symmetrical T-type is employed as the primary side compensation network. The resonance condition of the parameters for the equivalent circuit are given by

$$
\begin{gathered}
j \omega L_{1}+\frac{1}{j \omega C_{1}}=0 \\
j \omega L_{P}+\frac{1}{j \omega C_{1}}+\frac{1}{j \omega C_{2}}=0
\end{gathered}
$$

From (4) and (6), the input impedance $Z_{i n}$ and the current $I_{p}$ at the resonance condition can be derived as

$$
\begin{gathered}
Z_{\text {in }}=\frac{\left(\omega L_{1}\right)^{2}}{Z_{r}} \\
I_{p}=\frac{U_{\text {in }}}{j \omega L_{1}}
\end{gathered}
$$

It can be seen that the input impedance is pure resistance when $Z_{r}$ is resistive. Zero phase angle (ZPA) between output voltage and output current of the HFI can be achieved. The current $I_{p}$ of the primary transmitting coil is only related to the input voltage $U_{\text {in }}$ and $L_{1}$ with the load-independent characteristic. In order to reduce the communication link between the primary and the secondary side, the primary side adopts symmetrical LCC compensation network to provide a constant voltage source for the receiving coil and the induced voltage of receiving coil is

$$
U_{S}=j \omega M I_{p}
$$


Constant current output and constant voltage output is achieved by changing the topology of the secondary side. From Figure 2, there are three components in the T-type network besides the inductance of receiving coil. The output characteristics are different under asymmetric conditions. In order to get constant current output and constant voltage output, the impedances of $X_{C 3}, X_{C 4}$, and $X_{L 4}$ for the left side, the down side, and the right side in a general LCC compensation network are introduced here. The impedance of receiving coil $X_{L S}$ is set as reference quantity. Then the T-type provides three freedom degrees to adjust the transmission characteristic: $\lambda_{1}$ is the ratio of the capacitance $C_{3}$ in the left side to $X_{L S}, \lambda_{2}$ is the ratio of the capacitance $C_{4}$ in the down side to $X_{L S}$, and $\lambda_{3}$ is the ratio of the inductance $L_{4}$ in the right side to $X_{L S} . \lambda_{1}, \lambda_{2}$, and $\lambda_{3}$ are defined as follows:

$$
\lambda_{1}=\frac{X_{C 3}}{X_{L S}} \lambda_{2}=\frac{X_{C 4}}{X_{L S}} \lambda_{3}=\frac{X_{L 4}}{X_{L S}}
$$

Similar to the primary side, the receiver side equivalent impedance is

$$
\begin{aligned}
Z_{\mathrm{s}}=j \omega L_{s} & +\frac{1}{j \omega C_{3}}+\left(\frac{1}{j \omega C_{4}}\right)\left(j \omega L_{4}+R_{o}\right) \\
& =\frac{B_{1} X_{L S}+j B_{2} X_{L S} R_{O}}{j B_{3} X_{L S}+R_{O}}
\end{aligned}
$$

where

$$
B_{1}=\lambda_{2}-\lambda_{3}-\lambda_{1} \lambda_{2}+\lambda_{1} \lambda_{3}+\lambda_{2} \lambda_{3} \quad B_{2}=1-\lambda_{1}-\lambda_{2} \quad B_{3}=\lambda_{3}-\lambda_{2}
$$

The voltage and current of load are derived from the following formula:

$$
\begin{gathered}
I_{O}=\frac{U_{S}}{\left(1-\omega^{2} L_{3} C_{4}\right) R_{O}-j \omega\left(\omega^{2} L_{3} L_{4} C_{4}-L_{3}-L_{4}\right)} \\
U_{O}=\frac{U_{S} R_{O}}{\left(1-\omega^{2} L_{3} C_{4}\right) R_{O}-j \omega\left(\omega^{2} L_{3} L_{4} C_{4}-L_{3}-L_{4}\right)}
\end{gathered}
$$

By substituting (12) into (15) and (16), we have

$$
\begin{aligned}
I_{O} & =\frac{\lambda_{2} U_{S}}{\left(\lambda_{1}+\lambda_{2}-1\right) R_{O}-j\left(\lambda_{3}-\lambda_{2}+\lambda_{1} \lambda_{2}-\lambda_{1} \lambda_{3}-\lambda_{2} \lambda_{3}\right) X_{L S}} \\
U_{O} & =\frac{\lambda_{2} U_{S} R_{O}}{\left(\lambda_{1}+\lambda_{2}-1\right) R_{O}-j\left(\lambda_{3}-\lambda_{2}+\lambda_{1} \lambda_{2}-\lambda_{1} \lambda_{3}-\lambda_{2} \lambda_{3}\right) X_{L S}}
\end{aligned}
$$

To achieve constant current output (CC mode) independent of load, the coefficient of $R_{\mathrm{O}}$ in (17) should be equal to zero, and we get

$$
\lambda_{1}=1-\lambda_{2}
$$

The relationship between $C_{3}, C_{4}$, and $L_{S}$ in $C C$ mode can be expressed as

$$
j \omega L_{S}+\frac{1}{j \omega C_{3}}+\frac{1}{j \omega C_{4}}=0
$$

Then, the current $I_{\mathrm{O}}$ can be calculated as

$$
I_{O}=\frac{U_{S}}{j\left(1-\lambda_{1}\right) \omega L_{S}}=\frac{U_{S}}{j \lambda_{2} \omega L_{S}}
$$

From (21), the condition of constant current is also independent of the compensation inductance $\lambda_{3}$. Accordingly, by substituting (10) and (11) into (21), the ratio of the output current $I_{O}$ to the input voltage $U_{\text {in }}$, which is marked as the transconductance gain $G_{\mathrm{CC}}$, is derived as

$$
G_{C C}=\frac{M}{j\left(1-\lambda_{1}\right) \omega L_{1} L_{S}}
$$


Transconductance gain $G_{C C}$ is load-independent constant at a certain input voltage $U_{\text {in }}$ and a certain frequency $f$. It can be easily deduced that the CC mode can be implemented at this coefficient regardless of the variable load. The value of $Z_{S}$ can be simplified as

$$
Z_{\mathrm{s}}=\frac{\left(1-2 \lambda_{1}+\lambda_{1}^{2}\right) X_{L S}}{j\left(\lambda_{1}+\lambda_{3}-1\right) X_{L S}+R_{O}}
$$

Taking $\lambda_{2}=\lambda_{3}$, which means the down hand capacitor $C_{4}$ and the right-hand inductance $L_{4}$ is under resonant condition, the pure resistance of $Z_{S}$ can be realized and the solution can be obtained as follows:

$$
Z_{\mathrm{s}}=\frac{\left(1-2 \lambda_{1}+\lambda_{1}^{2}\right) X_{L S}}{R_{O}}
$$

To realize the constant voltage output ( $C V$ mode) against variable resistive load $R_{O}$, the coefficient of $X_{L S}$ in (18) should be set to zero, and we get

$$
\lambda_{3}=\frac{\left(\lambda_{1}-1\right) \lambda_{2}}{\lambda_{1}+\lambda_{2}-1}
$$

From (25), it can be seen that if the sum of $\lambda_{1}$ and $\lambda_{2}$ is equal to 1 , the $\lambda_{3}$ cannot be regulated and the CV mode cannot be realized. Therefore, the (20) cannot be maintained. The relationship between $C_{3}, C_{4}, L_{4}$, and $L_{S}$ in $C V$ mode can be expressed as

$$
\left(j \omega L_{S}+\frac{1}{j \omega C_{3}}\right)\left(j \omega L_{4}+\frac{1}{j \omega C_{4}}\right)-\frac{L_{4}}{C_{4}}=0
$$

Then, the output voltage $U o$ can be calculated as

$$
U_{O}=\frac{\lambda_{2} U_{S}}{\lambda_{1}+\lambda_{2}-1}
$$

The voltage gain $G_{C V}$ is given by substituting (10), (11) into (27) as

$$
G_{C V}=\frac{\lambda_{2} M}{\left(\lambda_{1}+\lambda_{2}-1\right) L_{1}}
$$

The coefficients of $B_{1}, B_{2}, B_{3}$ in (14) and the $Z_{\mathrm{S}}$ in (13) can be simplified as

$$
\begin{gathered}
B_{1}=0 \quad B_{2}=1-\lambda_{1}-\lambda_{2} \quad B_{3}=\frac{\lambda_{2}}{1-\lambda_{1}} \\
Z_{\mathrm{s}}=\frac{\left(1-\lambda_{1}-\lambda_{2}\right)\left(1-\lambda_{1}\right) X_{L S} R_{O}\left[\lambda_{2} X_{L S}+j\left(1-\lambda_{1}\right) R_{O}\right]}{\lambda_{2}^{2} X_{L S}^{2}+\left(1-\lambda_{1}\right)^{2} R_{O}^{2}}
\end{gathered}
$$

From (19), it can be seen that by reasonably configuring the parameters of $\lambda_{1}$ and $\lambda_{2}$, the load-independent constant current output can be achieved. The CC mode is independent of $L_{4}$ but $L_{4}$ has an effect on the receiver equivalent impedance. From (25), by reasonably configuring the parameters of $\lambda_{1}, \lambda_{2}$, and $\lambda_{3}$, the load-independent constant voltage output can be achieved, and the receiver equivalent impedance is related to these three variables.

\subsection{Parameters Analysis}

In order to analyze the influence of key parameters on the system characteristics and provide a theoretical basis for the reasonable configuration of parameters, the effects of $f, \lambda_{1}, \lambda_{2}$, and $\lambda_{3}$ on the output of IPT system were analyzed as follows. For the size of transmitting and receiving coils fixed in practical application, $L_{\mathrm{P}}$ and $L_{\mathrm{S}}$ were set as a reference value to design other parameters. In order 
to provide a constant voltage source for the secondary side, the symmetrical T-type compensation network is adopted in the primary side. The original parameters of the analysis are shown in Table 1.

Table 1. System specification and parameter values.

\begin{tabular}{cccc}
\hline Symbol & Value & Symbol & Value \\
\hline$L_{1}$ & $19.95 \mu \mathrm{H}$ & $L_{\mathrm{S}}$ & $147.6 \mu \mathrm{H}$ \\
$L_{\mathrm{P}}$ & $603.8 \mu \mathrm{H}$ & $L_{4}$ & $106.24 \mu \mathrm{H}$ \\
$C_{1}$ & $183.02 \mathrm{nF}$ & $\mathrm{C}_{3}$ & $80.94 \mathrm{nF}$ \\
$C_{2}$ & $5.94 \mathrm{nF}$ & $C_{4}$ & $33 \mathrm{nF}$ \\
$U_{\mathrm{i}}$ & $40 \mathrm{~V}$ & $R_{\mathrm{O}}$ & $40-100 \Omega$ \\
$M$ & $34.21 \mu \mathrm{H}$ & $f$ & $85 \mathrm{kHz}$ \\
\hline
\end{tabular}

From (20) and (26), the resonant frequency $f_{C C}$ in $C C$ mode and $f_{C V} C V$ mode can be derived as

$$
\begin{gathered}
f_{C C}=\frac{1}{2 \pi} \sqrt{\frac{C_{3}+C_{4}}{L_{S} C_{3} C_{4}}} \\
f_{C V}=\frac{1}{2 \pi} \sqrt{\frac{D_{1} \pm \sqrt{D_{1}-4 D_{2}}}{2 L_{S} L_{4}}}
\end{gathered}
$$

where

$$
D_{1}=\frac{L_{S}}{C_{4}}+\frac{L_{4}}{C_{3}}+\frac{L_{4}}{C_{4}} \quad D_{2}=\frac{L_{S} L_{4}}{C_{3} C_{4}}
$$

Figure 3 shows the constant current output and constant voltage output can be achieved under different parameters with specific resonant frequency. The output characteristics vary with $f$ for different loads, but at a specific resonant frequency, the output current or voltage are load independent. In Figure $3 a, \lambda_{1}, \lambda_{2}$, and $\lambda_{3}$ are set at $0.28,0.72$, and 0.72 , respectively. It can be seen that there is a resonant frequency at about $85 \mathrm{kHz}$ for CC mode and $115.6 / 54.5 \mathrm{kHz}$ for CV mode. The constant current output is $1.1 \mathrm{~A}$ and the constant voltage output is $54 \mathrm{~V}$ and $56 \mathrm{~V}$, respectively. Moreover, changing the value of inductor $L_{4}$ to make $X_{\mathrm{L} 4}=X_{\mathrm{C} 3}$, there are other resonant frequencies. CC mode at $85 \mathrm{kHz}$ with 2.8 A constant current output, and CV mode at $90.9 / 43.5 \mathrm{kHz}$ with $100 / 24 \mathrm{~V}$ constant voltage output. It is shown that different $L_{4}$ does not affect the resonant point of the CC mode. The resonant frequency to realize CV mode is different, with frequency bifurcation phenomenon. What is more, if the parameters are not carefully chosen, the outputs of the system are very sensitive to frequency. It is unfavourable to IPT system. This method needs to vary resonant frequency, which increases the cost of control $[9,17]$.

The inductance $L s$ is related to the design of the magnetic coupler, i.e., the receiving coil. Considering the transmission capacity and efficiency, the receiving coil is fixed and can not be designed too small. In order to reduce the size and cost of compensation network parameters, parameter $\lambda_{1}$ was introduced. The current output curve versus $\lambda_{1}$ with different loads in DLCC compensation network is shown in Figure 4. For a given $\lambda_{2}$, there is a $\lambda_{1}$ so that the output current is independent of the load, as shown in Figure 4. When $\lambda_{2}$ equals 0.72 and $\lambda_{1}$ equals 0.28 , the constant current output is $1.48 \mathrm{~A}$. The larger deviation of $\lambda_{1}$ from 0.28 , the more obvious the change of current with load, and the smaller the current is. When $\lambda_{2}$ equals 0.28 and $\lambda_{1}$ equals 0.72 , the constant output current is $9 \mathrm{~A}$. With the deviation increase of $\lambda_{1}$, the current output has the same trend, but the stability is worse. It is shown that with the specific resonant frequency and source voltage, the constant current output is proportional to $\lambda_{1}$ and is inversely proportional to $\lambda_{2}$.

Figure 5 shows the current output curve versus the different normalized parameters of $\lambda_{1}, \lambda_{2}$, and $\lambda_{3}$ with different load conditions in CC mode. The four subgraphs in Figure 5 correspond to $20 \Omega$, $40 \Omega, 60 \Omega$, and $80 \Omega$, respectively. The sensitivity of parameters to the output current was studied. It can be seen from the brown dotted line in Figure 5 that the current output is independent of $\lambda_{3}$. Under different loads, the variation of $\lambda_{1}$ has a relatively small influence on current output and it is not 
sensitive. With the increase of load, the sensitivity of output current to $\lambda_{2}$ decreases and $\lambda_{1}$ increases. However, the sensitivity of $\lambda_{2}$ is greater than that of $\lambda_{1}$ overall, obviously.

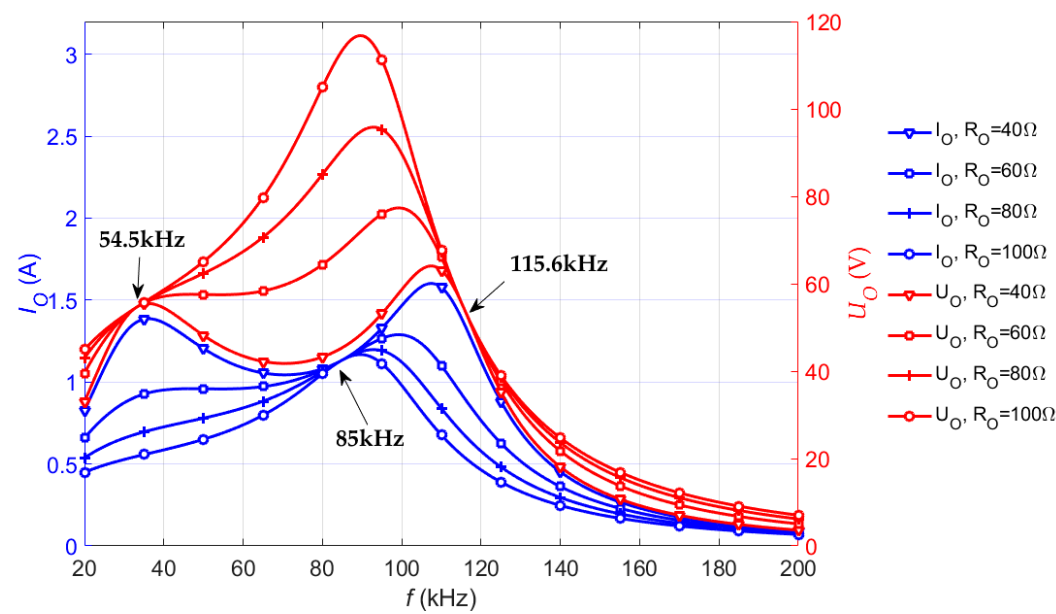

(a)

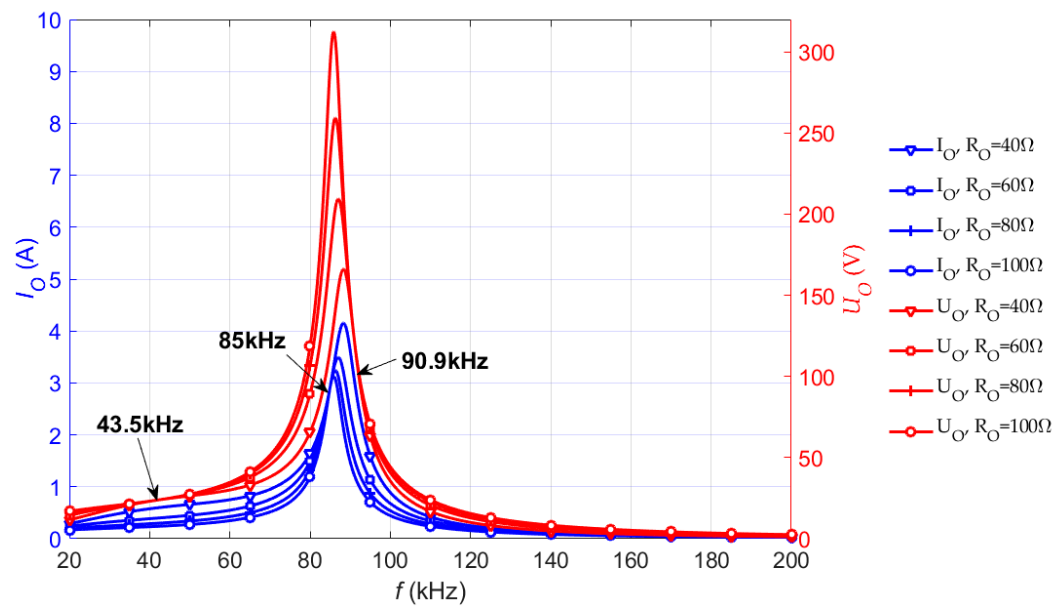

(b)

Figure 3. Relationship between $I \mathrm{O}, U_{\mathrm{O}}$ and $f$. (a) $\lambda_{3}=\lambda_{2}=1-\lambda_{1}=0.72 ;$ (b) $\lambda_{3}=\lambda_{2}=1-\lambda_{1}=0.28$.

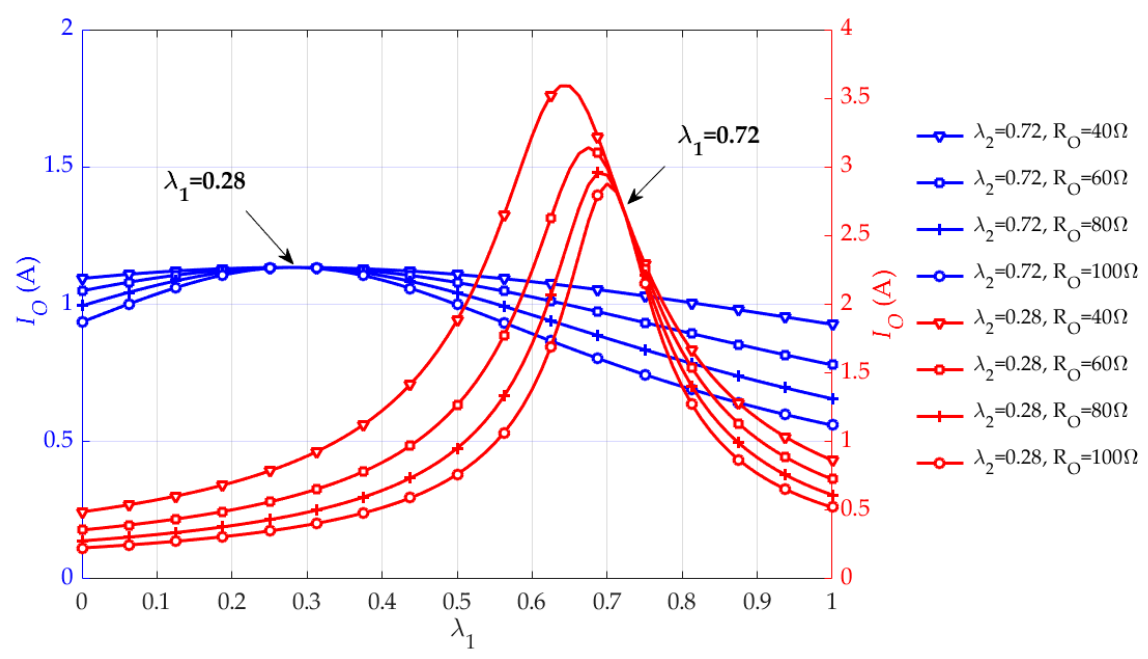

Figure 4. Relationship between $I_{\mathrm{O}}$ and $\lambda_{1}, \lambda_{2}$. 

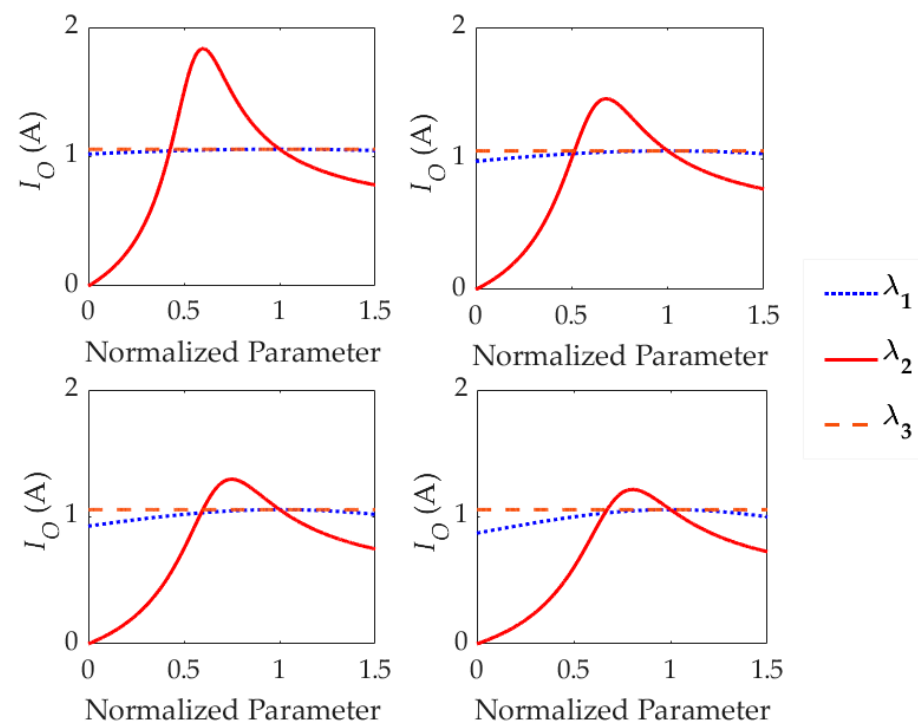

Figure 5. $I_{\mathrm{O}}$ versus normalized parameters.

The voltage output curve versus $\lambda_{2}$ with different loads is shown in Figure 6. It is shown that different parameters can achieve different load-independent constant voltage outputs. The same load-independent constant voltage output can also be achieved by different parameters. When the output voltage is set to $64 \mathrm{~V}$, there are two sets of implementation parameters, i.e., $\lambda_{1}=0.28, \lambda_{2}=0.36$, $\lambda_{3}=0.72$, and $\lambda_{1}=0.44 \lambda_{2}=0.28, \lambda_{3}=0.56$. When the output voltage is set to $100 \mathrm{~V}$, it can be achieved by parameters $\lambda_{1}=0.54 \lambda_{2}=0.28, \lambda_{3}=0.72$. Figure 7 shows constant voltage output capability under different parameters. With the increase of $\lambda_{1}$, the output voltage capacity becomes larger, but $\lambda_{1}$ is limited to less than 0.72 . The smaller the $\lambda_{1}$ is, the smaller the sensitivity of output voltage $U_{\mathrm{O}}$ to parameter $\lambda_{1}$ is and the more stable it is. Under the same condition of $\lambda_{1}$, the output capacity increases with the increase of $\lambda_{2}$. With the increase of $\lambda_{2}$, the starting voltage satisfying CV mode increases, and the range of $\lambda_{1}$ satisfying constant voltage decreases. It cannot meet the requirements of low constant voltage output.

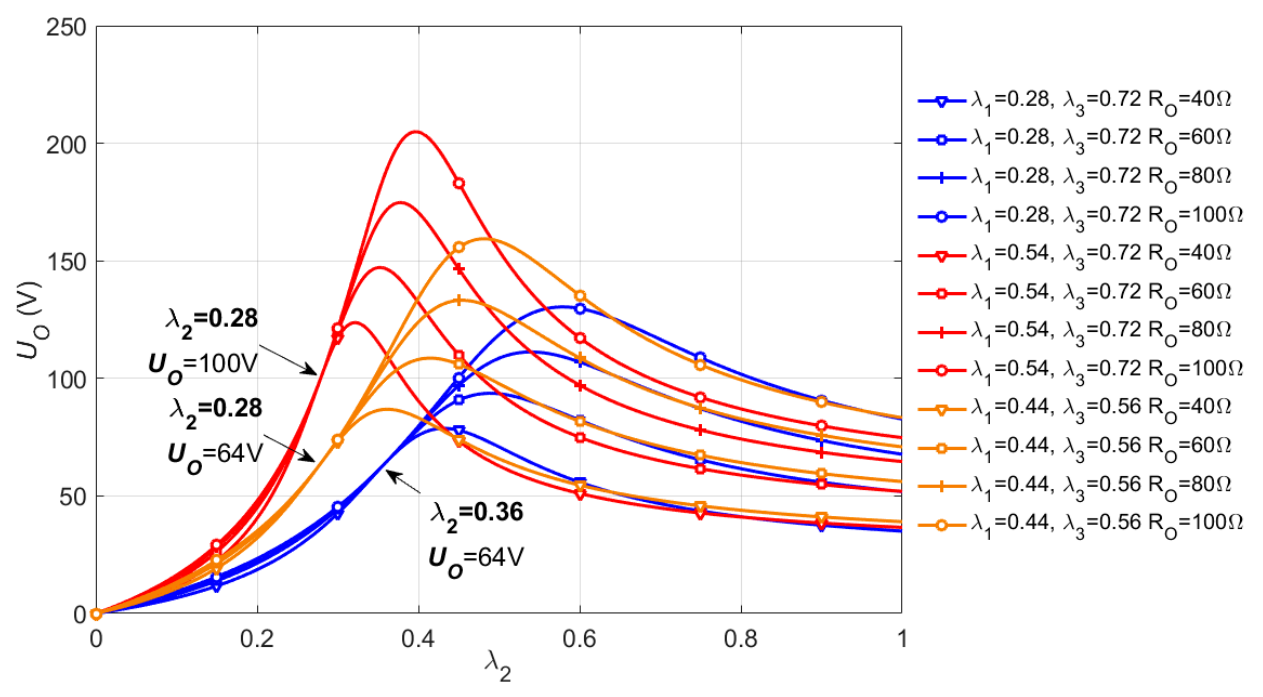

Figure 6. Relationship between of $U_{\mathrm{O}}$ and $\lambda_{1}, \lambda_{2}$. 


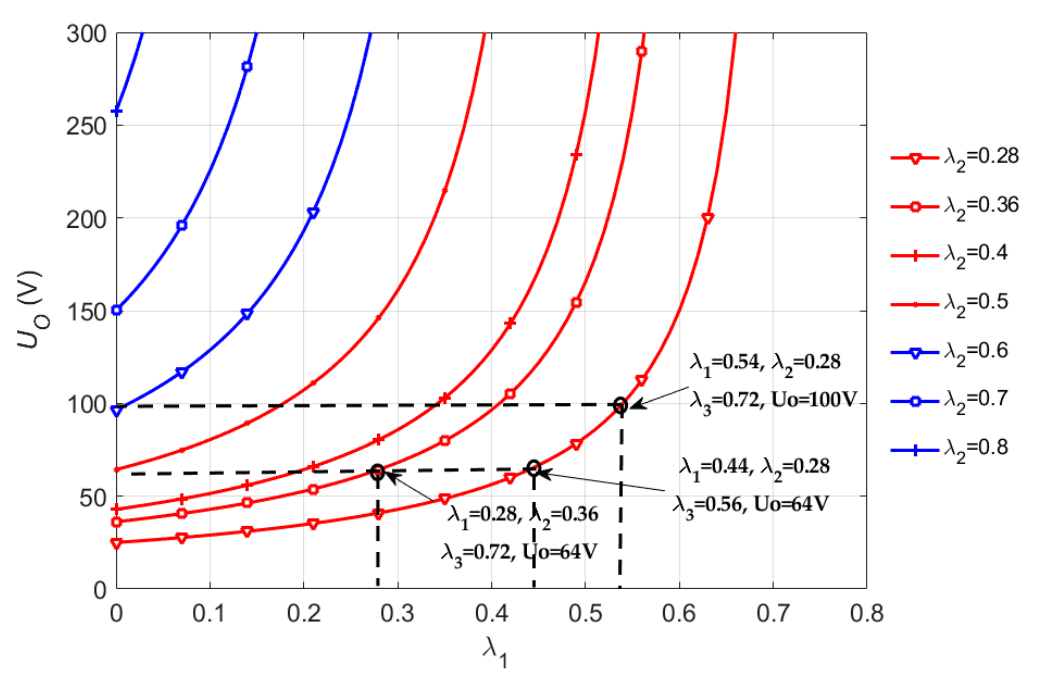

Figure 7. $U_{\mathrm{O}}$ versus $\lambda_{1}, \lambda_{2}$ in $\mathrm{CV}$ mode.

Taking into account the primary side, the input phase angle between the output current $I_{\text {in }}$ and voltage $U_{\text {in }}$ of the HFI is the main concern and is analyzed in the following. For the DLCC compensation network, the input impedances of HFI are different in CC and CV modes for different values of $\lambda_{1}$ and $\lambda_{2}$. The receiver equivalent impedance $Z_{S}$ can be calculated by (23) in CC mode and (30) in CV mode. By substituting (23) and (30) into (9), the input impedances $Z_{C C}$ in CC mode and $Z_{C V}$ in $\mathrm{CV}$ mode can be obtained as

$$
\begin{gathered}
Z_{\mathrm{CC}}=\left(\frac{L_{1}}{M}\right)^{2} \frac{\left(1-2 \lambda_{1}+\lambda_{1}^{2}\right) X_{L S}}{j\left(\lambda_{1}+\lambda_{3}-1\right) X_{L S}+R_{O}} \\
Z_{\mathrm{CV}}=\left(\frac{L_{1}}{M}\right)^{2} \frac{\left(1-\lambda_{1}-\lambda_{2}\right)\left(1-\lambda_{1}\right) X_{L S} R_{O}\left[\lambda_{2} X_{L S}+j\left(1-\lambda_{1}\right) R_{O}\right]}{\lambda_{2}^{2} X_{L S}^{2}+\left(1-\lambda_{1}\right)^{2} R_{O}^{2}}
\end{gathered}
$$

The input phase angle $\theta_{i}$ can be derived as

$$
\theta_{C C / V}=\frac{180^{\circ}}{\pi} \arctan \left[\frac{\operatorname{Im}\left(Z_{C C / V}\right)}{\operatorname{Re}\left(Z_{C C / V}\right)}\right]
$$

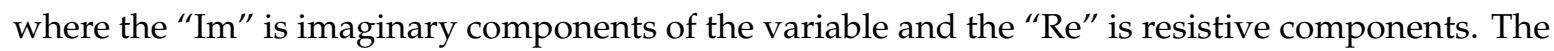
results of the input phase angle in CC mode $\left(\theta_{C C}\right)$ and $C V$ mode $\left(\theta_{C V}\right)$ are shown in Figures 8 and 9.

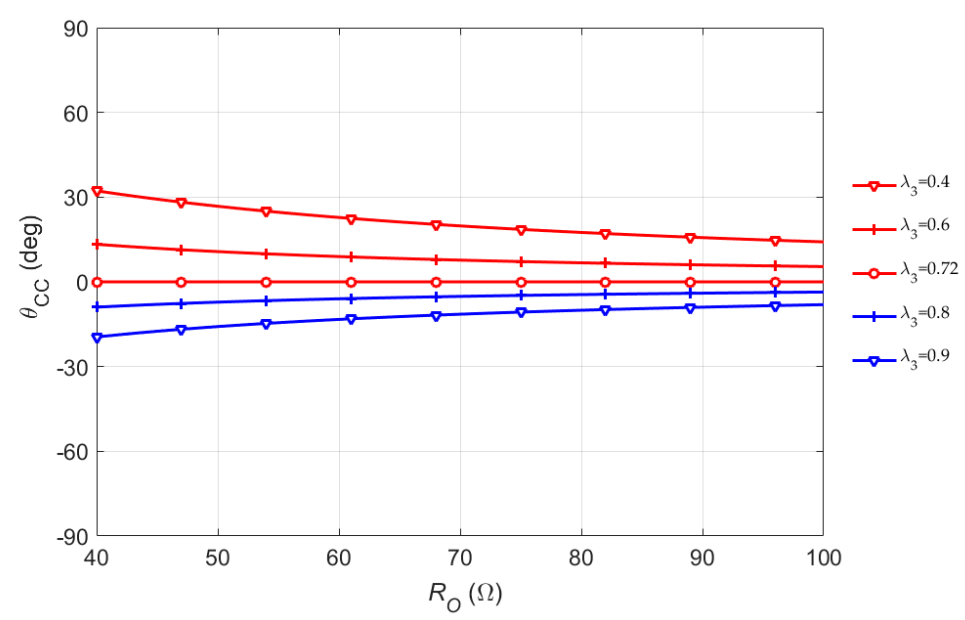

Figure 8. Phase angle of input impedance in CC mode. 


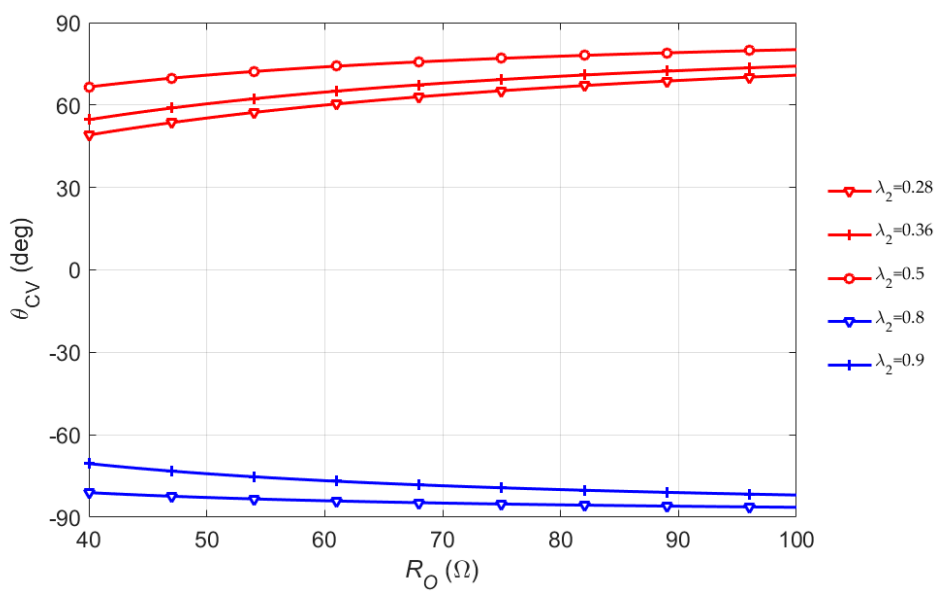

Figure 9. Phase angle of input impedance in CV mode.

According to (24), the constant current output can make the secondary impedance pure resistance by the change of $\lambda_{3}$. From Figure 8 , when $\lambda_{3}$ equals 0.72 (marked as $\lambda_{3 \_}$), no matter what the values of $\lambda_{1}$ and $\lambda_{2}$ are, the input impedance is pure resistance, which means no reactive power in the IPT system. The input impedance of the HFI is inductive when $\lambda_{3}$ is less than $\lambda_{3 \_} \mathrm{R}$ and capacitive when $\lambda_{3}$ is greater than $\lambda_{3 \_}$. Additionally, it is also shown that the phase angle decreases with the increase of load.

The phase angle of the IPT system in CV mode is shown in Figure 9. The input impedance cannot be pure resistance. When $\lambda_{2}$ equals 0.72 (marked as $\lambda_{2 \_}$), the phase difference is 90 degrees. The input impedance of the HFI is inductive when $\lambda_{2}$ is less than $\lambda_{2 \_} \mathrm{R}$ and capacitive when $\lambda_{2}$ is greater than $\lambda_{2 \_}$. The larger the difference between $\lambda_{2}$ and $\lambda_{2 \_}$, the smaller phase angle between the output current $I_{\text {in }}$ and voltage $U_{\text {in }}$ of the HFI. Hard switching will occur and the switching loss will increase if the input impedance of the IPT system is capacitive. Therefore, $\lambda_{2}$ less than $\lambda_{2 \_} \mathrm{R}$ is adopted in practical application.

According to the transconductance gain $G_{C C}$ (22) and the voltage gain $G_{C V}$ (28), the relationship between battery charging current, charging voltage, and source voltage of IPT system can be expressed as

$$
\begin{gathered}
G_{B C}=\frac{8}{\pi^{2}} G_{C C}=\frac{8}{\pi^{2}} \frac{M}{j\left(1-\lambda_{1}\right) \omega L_{1} L_{S}} \\
G_{B V}=G_{C V}=\frac{\lambda_{2} M}{\left(\lambda_{1}+\lambda_{2}-1\right) L_{1}}
\end{gathered}
$$

The current output in CC mode and voltage output in CV mode are related to the output voltage of HFI under the condition of fixed parameters. By adjusting the output voltage of HFI, the output voltage and current can be controlled, realizing the charging target of multi-voltage and current levels.

\section{Parameters Design}

From the above analysis, the constant voltage output and constant current output can be realized by configuring different parameters using DLCC compensation. The parameter $\lambda_{1}$ related to $C_{3}$ and the parameter $\lambda_{2}$ related to $C_{4}$ for achieving constant current output and constant voltage output were studied, respectively. For a battery that needs continuous charging, it is necessary to take into account the smooth conversion of charging mode. Assume the charging voltage and current of the battery are $U_{\mathrm{B}}$ and $I_{\mathrm{B}}$. At the particular moment $t$, the charging mode is switched from CC mode to CV mode, and the switching load is $R_{O}$. The voltage of the $R_{O}$ in CC mode at time $t_{-}$is marked as $U_{\mathrm{CC}}\left(t_{-}\right)$, and the voltage in $\mathrm{CV}$ mode at time $t_{+}$is marked as $U_{\mathrm{CV}}\left(t_{+}\right)$. There are two ways to change the charging mode 
by changing one parameter. With the constant input voltage, the voltages of batteries in CC mode and $\mathrm{CV}$ mode are derived, respectively, as

$$
\begin{gathered}
U_{C C}\left(t_{-}\right)=I\left(t_{-}\right) R_{O}^{\prime}=\frac{U_{S} R_{O}^{\prime}}{\left(1-\lambda_{1}\right) \omega L_{S}}=\frac{U_{S} R_{O}^{\prime}}{\lambda_{2} \omega L_{S}} \\
U_{C V}\left(t_{+}\right)=\frac{\lambda_{2} U_{S}}{\lambda_{1}+\lambda_{2}-1}=\frac{\lambda_{3} U_{S}}{1-\lambda_{1}}=\left(1-\frac{\lambda_{3}}{\lambda_{2}}\right) U_{S}
\end{gathered}
$$

where

$$
R_{O}^{\prime}=\frac{8}{\pi^{2}} \frac{U_{B}}{I_{B}}
$$

During switching operation, $U_{\mathrm{CC}}\left(t_{-}\right)$should be equal to $U_{\mathrm{CV}}\left(t_{+}\right)$. If it is achieved by changing $\lambda_{2}$, the parameters are related by

$$
\frac{U_{S} R_{O}^{\prime}}{\left(1-\lambda_{1}\right) \omega L_{S}}=\frac{\lambda_{3} U_{S}}{1-\lambda_{1}}
$$

The parameter $\lambda_{3}$ can be obtained as

$$
\lambda_{3}=\frac{8}{\pi^{2}} \frac{U_{B}}{\omega L_{S} I_{B}}
$$

Therefore, for a given battery known as constant charging voltage $U_{B}$ and the constant charging current $I_{B}$, the right-hand compensation inductor $L_{4}$ can be derived firstly by changing $\lambda_{2}$ as

$$
L_{4}=\frac{8}{\pi^{2}} \frac{U_{B}}{\omega I_{B}}
$$

According to (2) and (21), the value of $\lambda_{1}$ can be derived by

$$
\lambda_{1}=1-\frac{2 \sqrt{2}}{\pi} \frac{U_{S}}{\omega L_{S} I_{B}}
$$

After we get $\lambda_{1}$ and $\lambda_{3}$, the parameter $\lambda_{2}$ for CC mode and $\lambda_{2}$ can be given directly by solving (19) and (25) as

$$
\begin{gathered}
\lambda_{2 \_C C}=1-\lambda_{1} \\
\lambda_{2 \_C V}=\frac{\left(\lambda_{1}-1\right) \lambda_{3}}{\lambda_{1}-\lambda_{3}-1}
\end{gathered}
$$

If the charging profile is achieved by changing $\lambda_{1}$, the value of $\lambda_{3}$ should be chosen according to

$$
\frac{U_{S} R_{O}^{\prime}}{\lambda_{2} \omega L_{S}}=\left(1-\frac{\lambda_{3}}{\lambda_{2}}\right) U_{S}
$$

By solving (48), the parameter $\lambda_{3}$ is given by

$$
\lambda_{3}=\lambda_{2}+\frac{8}{\pi^{2}} \frac{U_{B}}{\omega L_{S} I_{B}}
$$

From (49), $\lambda_{2}$ should be calculated firstly by solving (26)

$$
\lambda_{2}=\frac{2 \sqrt{2}}{\pi} \frac{U_{S}}{\omega L_{S} I_{B}}
$$


By substituting (50) into (49), $L_{4}$ is given as following

$$
L_{4}=\frac{2 \sqrt{2} U_{S}+\frac{8}{\pi^{2}} U_{B}}{\omega I_{B}}
$$

The parameter $\lambda_{1}$ for CC mode and $\lambda_{2}$ can be given by solving (19) and (25) as

$$
\begin{gathered}
\lambda_{1 \_C C}=1-\lambda_{2} \\
\lambda_{1 \_C V}=1+\frac{\lambda_{2} \lambda_{3}}{\lambda_{2}-\lambda_{3}}
\end{gathered}
$$

After we get $\lambda_{1}$ and $\lambda_{2}$, the corresponding compensation capacitance $C_{3}$ and $C_{4}$ can be obtained by solving (12). A design method of DLCC compensated IPT system is proposed in this section to realize $\mathrm{CC}$ output and $\mathrm{CV}$ output. For a given input voltage and an output charging profile, two methods with different $C_{3}, C_{4}$, and $L_{4}$ can be obtained by the following flowchart shown in Figure 10 .

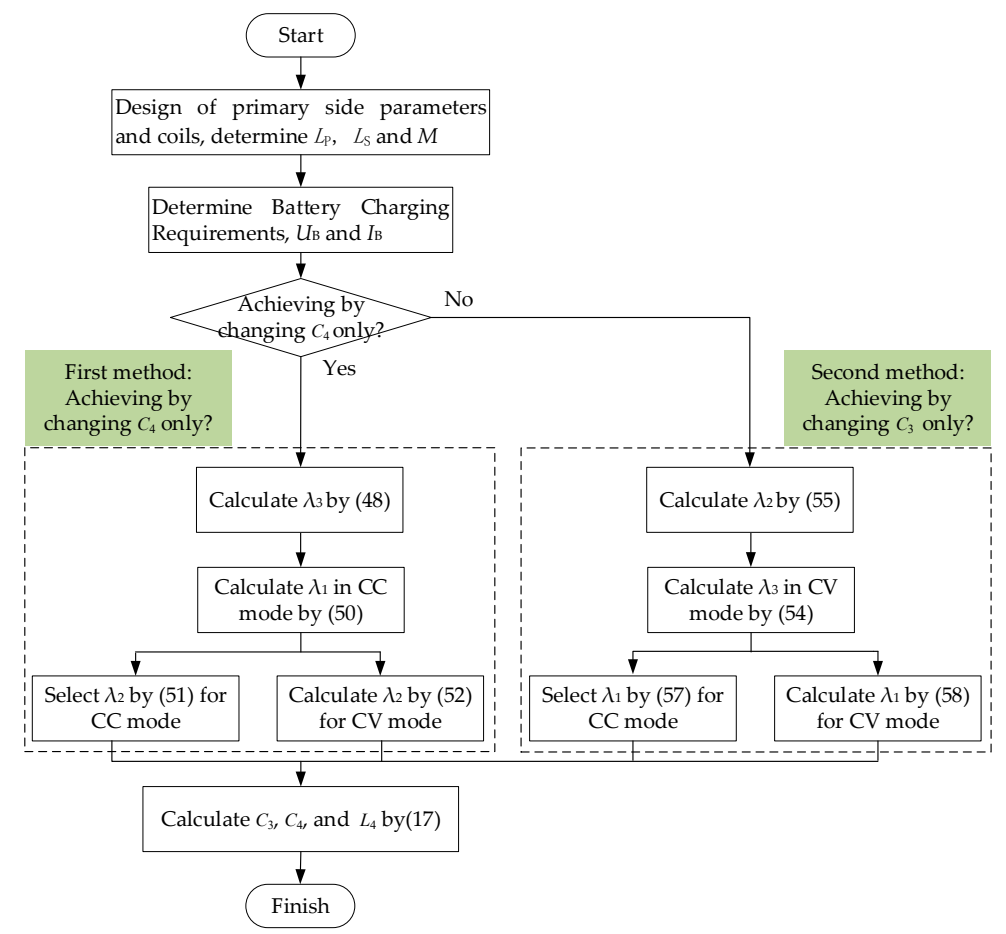

Figure 10. Flowchart of parameters design.

According to the comprehensive analysis of the parameter characteristics in Section 2, more than one set of parameters can be achieved for a specific output voltage in the CV mode. As shown in Figure 10, two methods achieve constant voltage output by changing the capacitance parameters: the parallel capacitance of $C^{\prime}{ }_{4}$ or $C_{3}{ }^{\prime}$. The left branch of the flowchart is the first method by changing $C_{4}$ only, and the right branch is the second method by changing $C_{3}$ only. The two methods are implemented in different ways and require different components. The first method requires an additional switch and an auxiliary capacitor to realize the change from CC mode to CV mode. The $\lambda_{3}$ of CC mode makes the IPT system realize ZPA. From (21), it can be seen that the value of $\lambda_{3}$ in CC mode does not affect the constant current output. If the ZPA of the IPT system is not considered, $\lambda_{3}$ and $\lambda_{2}$ can be calculated in CV mode firstly, and change $\lambda_{1}$ to achieve the conversion between the two modes by an auxiliary capacitor and an additional switch. However, this method increases the reactive power of the system. To realize ZPA in CC mode by changing $\lambda_{1}, \lambda_{3}$ also needs to be changed at the same time. Comparing with the above two methods, two parameters in the $\mathrm{CV}$ mode have changed, i.e., two 
additional switches and an auxiliary capacitor and an auxiliary inductor are needed. This method is not adopted for the increasing of cost and complexity.

The input voltage $U_{\mathrm{d}}$ is $40 \mathrm{~V}$ and the operating frequency of IPT system set to $85 \mathrm{kHz}$. For a given receiving coil set to be $106.24 \mu \mathrm{H}$, two sets (two for each set) of parameters were designed according to Figure 11. The values of inductance and capacitance are listed in Tables 2 and 3. Both methods need an additional switch operation to change from CC mode to CV mode, but the first method can realize ZPA in CC mode. From the analysis of the parameters in the Section 2, it can be seen that the stability of the system is affected by the sensitivity of different parameters to operational frequency and parameter offset. It can be seen from Figures 3 and 4 that the frequency offset and parameter offset has less influence on the current output when $\lambda_{1}=0.28$. From Figure 7 , it can be seen that smaller $\lambda_{1}$ is beneficial to the stability of constant voltage output. Consequently, the next part of the experimental platform adopts the first method.

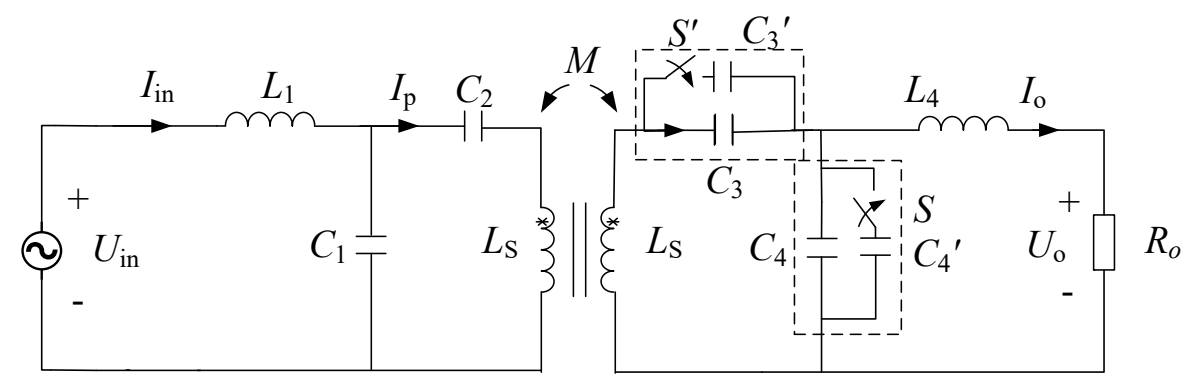

Figure 11. Two methods of charging mode conversion.

Table 2. Theoretically calculated parameter values of first method.

\begin{tabular}{cccc}
\hline$\lambda_{\mathbf{1}}$ & $\boldsymbol{\lambda}_{\mathbf{2}}$ for CC Mode & $\boldsymbol{\lambda}_{\mathbf{2}}$ for CV Mode & $\boldsymbol{\lambda}_{\mathbf{3}}$ \\
\hline 0.28 & 0.72 & 0.36 & 0.72 \\
\hline$C_{3}$ & $C_{4}$ & $C_{4}$ & $L_{4}$ \\
$80.94 \mathrm{nF}$ & $33 \mathrm{nF}$ & $66 \mathrm{nF}$ & $106.24 \mu \mathrm{H}$ \\
\hline
\end{tabular}

Table 3. Theoretically calculated parameter values of second method.

\begin{tabular}{cccc}
\hline $\boldsymbol{\lambda}_{\mathbf{1}}$ for CC Mode & $\boldsymbol{\lambda}_{\mathbf{1}}$ for CV Mode & $\boldsymbol{\lambda}_{\mathbf{2}}$ & $\boldsymbol{\lambda}_{\mathbf{3}}$ \\
\hline 0.72 & 0.44 & 0.28 & 0.56 \\
\hline$C_{3}$ & $C_{3}$ & $C_{4}$ & $L_{4}$ \\
$33 \mathrm{nF}$ & $51.51 \mathrm{nF}$ & $80.94 \mathrm{nF}$ & $82.63 \mu \mathrm{H}$ \\
\hline
\end{tabular}

\section{Experimental Verification}

To verify the analysis above, an experimental prototype shown in Figure 12 was built. The value of $L_{4}$ can be directly calculated by battery parameters and is independent of other parameters through the first method, as shown in Figure 10. So, the experimental platform is set up according to Figure 11. The parameters of the compensation networks are provided in Table 2. The dc input voltage $U_{d}$ is $40 \mathrm{~V}$. The primary and secondary coils are both round structures, the diameters are $75 \mathrm{~mm}$ and $35 \mathrm{~mm}$, respectively. The air gap is set to $8 \mathrm{~mm}$. Switches $S_{1,2,3,4}$ of full bridge inverter are IPW90R120C 3 and secondary rectifier diodes are DSEI $2 \times 101$. The output filter uses $C_{f}$ of $560 \mu \mathrm{F}$. The internal resistance of battery can be equivalent to the non-linear resistance $[15,16]$. In this experiment, an electronic load with wide range was used to simulate the equivalent behavior of batteries during charging. For simplicity, the whole charging profile is set to two sections: charging current $I_{\mathrm{B}} 1 \mathrm{~A}$ and charging voltage $U_{\mathrm{B}} 64 \mathrm{~V}$. 


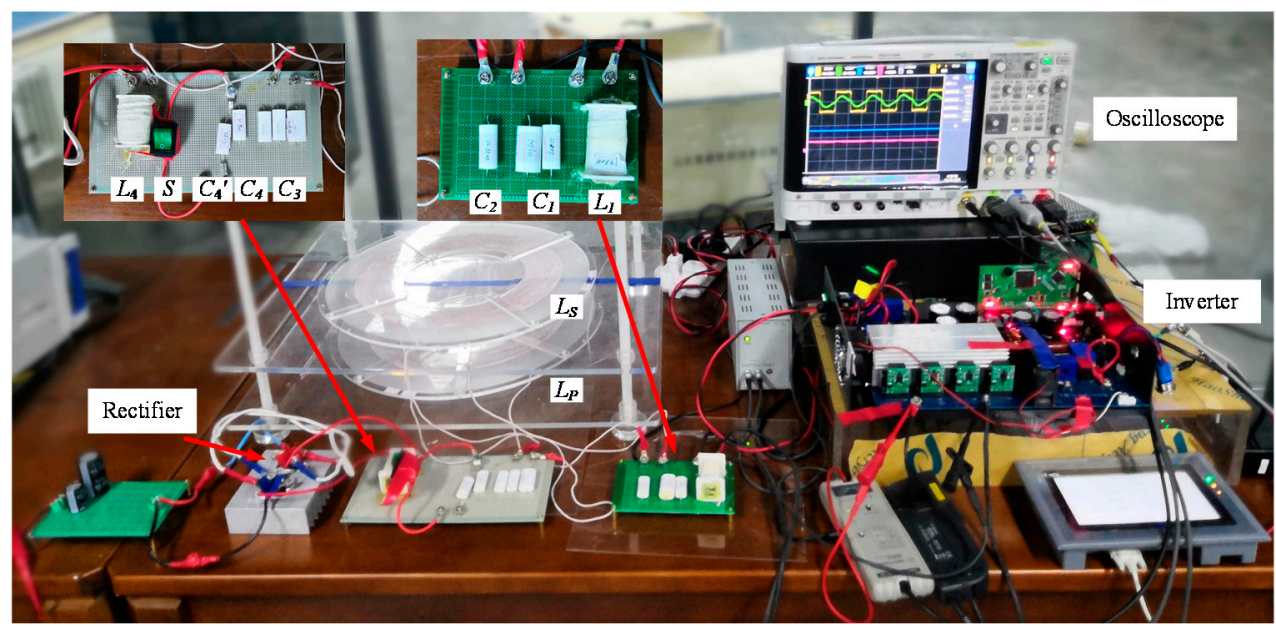

Figure 12. Prototype of the WPT charging system.

Firstly, the results of CC mode were analyzed. With different load resistance, the output waveforms $u_{\text {in }}, i_{\text {in }}$ of the inverter, and the current $i_{\mathrm{P}}$ are shown in Figure 13 . The transient waveforms $u_{\mathrm{B}}$ and $i_{\mathrm{B}}$ with load resistance switching from $40 \Omega$ to $60 \Omega$ are shown in Figure 14. It can be seen that under the symmetrical T-type compensation parameters, the waveform of $i_{\text {in }}$ is always nearly in phase with $u_{\text {in }}$. Nearly zero reactive power is generated in this case of well compensation. The current flowing through the transmitting coil is independent of the load. In Figure 14, the charging current $I_{\mathrm{B}}$ of IPT is $1.0 \mathrm{~A}$ at $R_{\mathrm{B}}=40 \Omega$ and $994 \mathrm{~mA}$ at $60 \Omega$. The fluctuation margin of charging current is less than $1 \%$ and the charging process is basically constant. After the circuit is stabilized, the load voltage increases with the increase of the load.

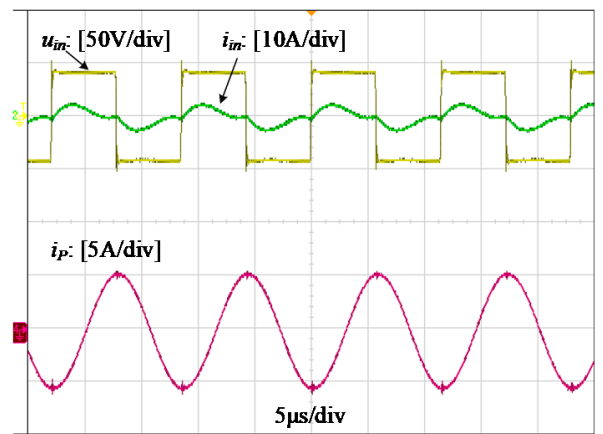

(a)

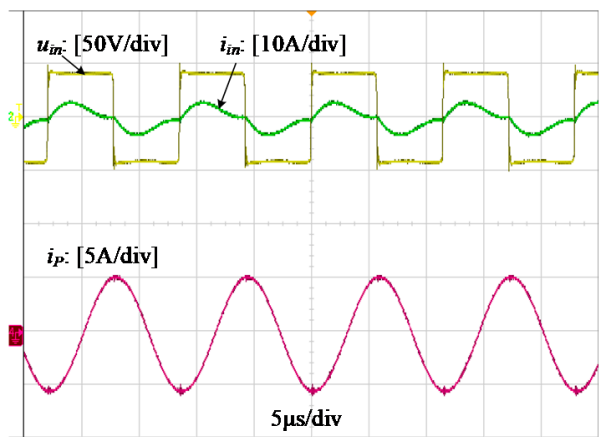

(b)

Figure 13. Experimental waveforms of $u_{\mathrm{in}}, i_{\mathrm{in}}$, and $i_{\mathrm{P}}$ in CC mode when $(\mathbf{a}) R_{\mathrm{B}}=40 \Omega$ and $(\mathbf{b}) R_{\mathrm{B}}=60 \Omega$.

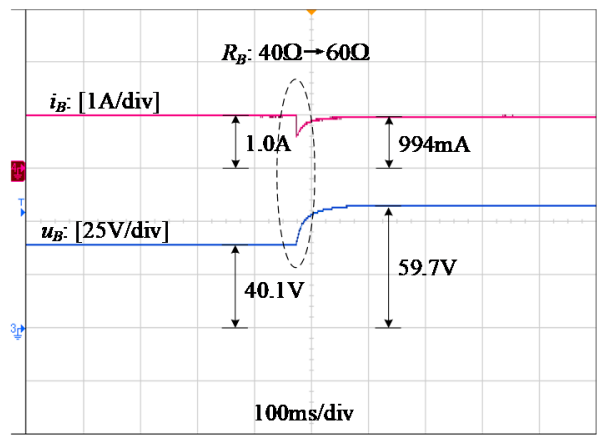

Figure 14. Transient waveforms switch from $40 \Omega$ to $60 \Omega$ at CC mode. 
The results of CV mode are shown in Figures 15 and 16. As shown in Figure 11, the IPT system operates in $\mathrm{CV}$ mode when switch $\mathrm{S}$ is $\mathrm{ON}$, in which the capacitor changes from $C_{4}$ to $C_{4} \mathrm{CV}$ by paralleled connection of $C_{4}{ }^{\prime}$ to $C_{4}$. The value of equivalent capacitor is given as

$$
\mathrm{C}_{4} \mathrm{CV}_{\mathrm{C}}=\mathrm{C}_{4}+\mathrm{C}_{4}^{\prime}
$$

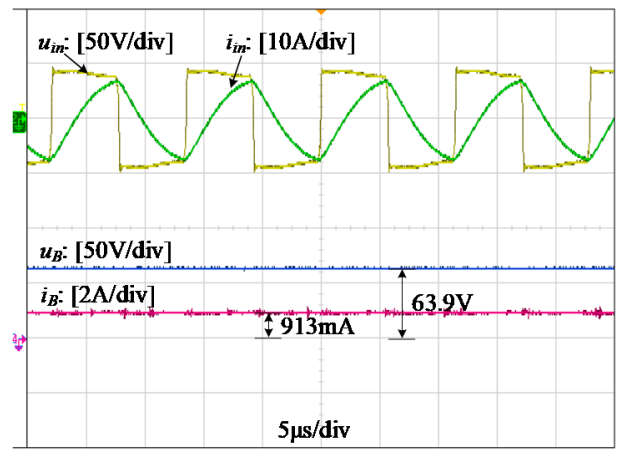

(a)

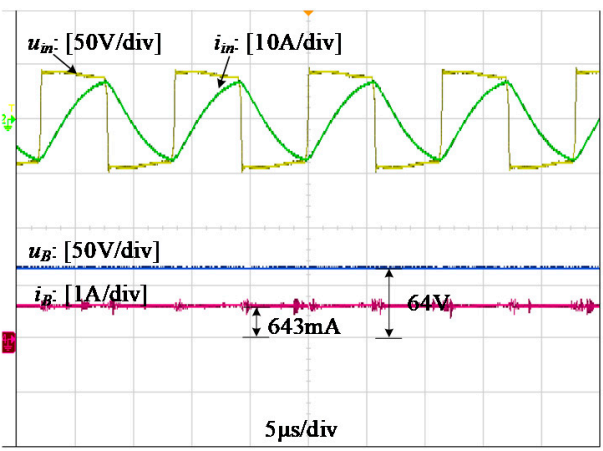

(b)

Figure 15. Experimental waveforms of $u_{\text {in }}, i_{\text {in }}$ in CV mode when $(\mathbf{a}) R_{\mathrm{B}}=70 \Omega$ and (b) $R_{\mathrm{B}}=100 \Omega$.

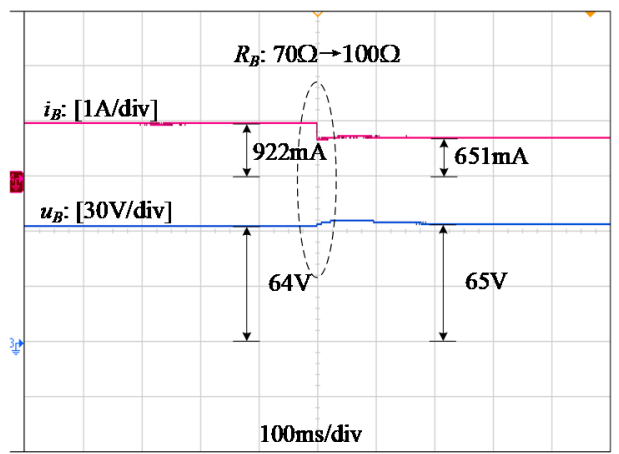

Figure 16. Transient waveforms from $70 \Omega$ switch to $100 \Omega$ at CV mode.

The capacitance $C_{4}$ changes from $33 \mathrm{uH}$ to $66 \mathrm{uH}$. Figure 15 shows the output waveforms $u_{\text {in }}, i_{\text {in }}$ of the inverter, and the output waveforms $u_{\mathrm{B}}, i_{\mathrm{B}}$ of load under different resistance. The waveform of $i_{\text {in }}$ lags behind $u_{\mathrm{in}}$, which illustrates that IPT is charging with reactive power at CV mode. In Figure 16, the charging voltage $u_{\mathrm{B}}$ of IPT system is $63.9 \mathrm{~V}$ at $R_{\mathrm{B}}=70 \Omega$ and $64 \mathrm{~V}$ at $100 \Omega$. It remains constant after the circuit is stabilized, and the load current decreases with the increase of the load. The fluctuation margin of charging voltage is less than $1 \%$ and the charging process is basically constant.

Figure 17 shows the charging mode changing from CC mode to $C V$ mode, while the battery voltage $u_{B}$ and current $i_{B}$ change smoothly during the mode transition. In CC mode, the charging current $i_{B}$ varies from $1.11 \mathrm{~A}$ at $R_{B}=40 \Omega$ to $1.09 \mathrm{~A}$ at $R_{B}=64 \Omega$, and the fluctuation margin of $i_{B}$ is $1.8 \%$. The charging voltage $u_{B}$ varies from $63.5 \mathrm{~V}$ at $R_{B}=67 \Omega$ to $64.9 \mathrm{~V}$ at $R_{B}=100 \Omega$, and the fluctuation margin of $u_{B}$ is less than $2.5 \%$. The whole charging profile, including charging current $I_{B}$ and charging voltage $U_{B}$ versus $R_{B}$, is shown in Figure 18. At the beginning of charging, the battery current is maintained at $1 \mathrm{~A}$. With the increase of the equivalent battery load, the battery voltage increases to the required $64 \mathrm{~V}$ and then remains stable. 


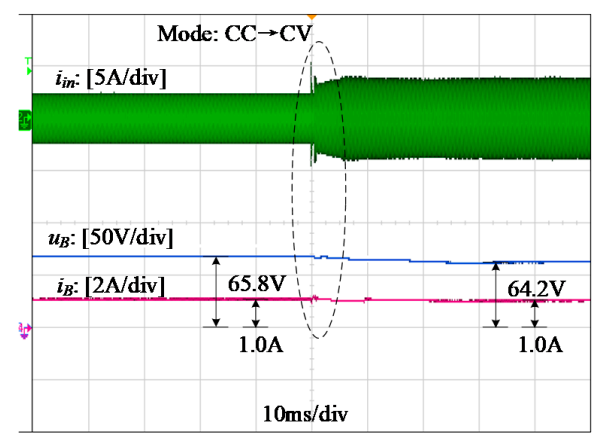

Figure 17. Transient waveforms of $i_{\mathrm{in}}, u_{\mathrm{B}}$ and $i_{\mathrm{B}}$ from CC mode to $\mathrm{CV}$ mode.

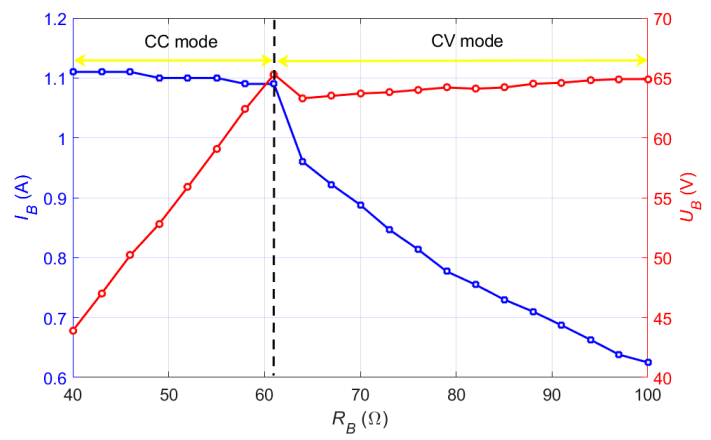

Figure 18. Measured charging profile versus $R_{\mathrm{B}}$.

\section{Conclusions}

In this study, a DLCC compensated IPT system was designed to achieve load independent constant current output and constant voltage output for battery charging applications. The characteristics of LCC compensate network with three design freedoms has been analyzed in detail. According to the theoretical analysis, two parametric design methods of realizing CC mode and CV mode have been proposed. With one switch and an additional capacitor, a smooth mode transition can be achieved. The fluctuation margins of charging current and charging voltage are both less than $2.5 \%$, which meets the charging requirements of batteries. The experimental results agree well with the performance of the proposed method which demonstrates that it is feasible to a given charging characteristics of battery.

Author Contributions: Writing—original draft, J.Y.; formal analysis, X.Y.; investigation, Q.L. and Y.S.; writing-review \& editing, X.Z.

Funding: This research was funded by the Fundamental Research Funds for the Central Universities, grant number E19JB500180.

Conflicts of Interest: The authors declare no conflict of interest.

\section{References}

1. RamRakhyani, A.K.; Mirabbasi, S.; Chiao, M. Design and optimization of resonance-based efficient wireless power delivery systems for biomedical implants. IEEE Trans. Biomed. Circuits Syst. 2011, 5, 48-63. [CrossRef] [PubMed]

2. Wu, R.; Li, W.; Luo, H.; Sin, J.K.; Yue, C.P. Design and characterization of wireless power links for brain-machine interface applications. IEEE Trans. Power Electron. 2014, 29, 5462-5471. [CrossRef]

3. Patil, D.; Mcdonough, M.K.; Miller, M.; Fahimi, B.; Balsara, P.T. Wireless Power Transfer for Vehicular Applications: Overview and Challenges. IEEE Trans. Transp. Electrifi. 2018, 4, 3-37. [CrossRef]

4. Li, S.; Mi, C.C. Wireless Power Transfer for Electric Vehicle Applications. IEEE J. Emerg. Sel. Topics Power Electron. 2015, 3, 4-17.

5. Buja, G.; Bertoluzzo, M.; Mude, K.N. Design and Experimentation of WPT Charger for Electric City Car. IEEE Trans. Ind. Electron. 2015, 62, 7436-7447. [CrossRef] 
6. Han, W.; Chau, K.T.; Zhang, Z. Flexible induction heating using magnetic resonant coupling. IEEE Trans. Ind. Electron. 2016, 64, 1982-1992. [CrossRef]

7. Han, H.; Mao, Z.; Zhu, Q.; Su, M.; Hu, A.P. A 3D Wireless Charging Cylinder with Stable Rotating Magnetic Field for Multi-Load Application. IEEE Access. 2019, 7, 35981-35997. [CrossRef]

8. Kang, X.U.; Chen, X.; Liu, D. Electrical Impedance Transformation Techniques for an Ultrasonic Coupling Wireless Power Transfer System under Sea Water. Proc. CSEE 2015, 35, 4461-4467.

9. Qu, X.; Chu, H.; Wong, S.C.; Chi, K.T. An IPT Battery Charger with Near Unity Power Factor and Load-independent Constant Output Combating Design Constraints of Input Voltage and Transformer Parameters. IEEE Trans. Power Electron. 2019, 34, 7719-7727. [CrossRef]

10. Yilmaz, M.; Krein, P.T. Review of Battery Charger Topologies, Charging Power Levels, and Infrastructure for Plug-In Electric and Hybrid Vehicles. IEEE Trans. Power Electron. 2013, 28, 2151-2169. [CrossRef]

11. Khaligh, A.; Li, Z. Battery, Ultracapacitor, Fuel Cell, and Hybrid Energy Storage Systems for Electric, Hybrid Electric, Fuel Cell, and Plug-In Hybrid Electric Vehicles: State of the Art. IEEE Trans. Veh. Technol. 2010, 59, 2806-2814. [CrossRef]

12. Li, T.; Wang, X.; Zheng, S.; Liu, C. An Efficient Topology for Wireless Power Transfer over a Wide Range of Loading Conditions. Energies 2018, 11, 141. [CrossRef]

13. Li, H.; Li, J.; Wang, K.; Chen, W.; Yang, X. A Maximum Efficiency Point Tracking Control Scheme for Wireless Power Transfer Systems Using Magnetic Resonant Coupling. IEEE Trans. Power Electron. 2015, 30, 3998-4008. [CrossRef]

14. Wu, H.H.; Gilchrist, A.; Sealy, K.D.; Bronson, D. A High Efficiency $5 \mathrm{~kW}$ Inductive Charger for EVs Using Dual Side Control. IEEE Trans. Ind. Electron. 2012, 8, 585-595. [CrossRef]

15. Li, Z.; Zhu, C.; Jiang, J.; Song, K. A 3 kW Wireless Power Transfer System for Sightseeing Car Supercapacitor Charge. IEEE Trans. Power Electron. 2017, 32, 3301-3316. [CrossRef]

16. Liu, N.; Habetler, T.G. Design of a Universal Inductive Charger for Multiple Electric Vehicle Models. IEEE Trans. Power Electron. 2015, 30, 6378-6390. [CrossRef]

17. Wang, C.S.; Covic, G.A.; Stielau, O.H. Power Transfer Capability and Bifurcation Phenomena of Loosely Coupled Inductive Power Transfer Systems. IEEE Trans. Ind. Electron. 2004, 51, 148-157. [CrossRef]

18. Auvigne, C.; Germano, P.; Ladas, D.; Perriard, Y. A Dual-topology ICPT Applied to an Electric Vehicle Battery Charger. In Proceedings of the 2012 International Conference on Electrical Machines, Marseille, France, 2-5 September 2012; pp. 2287-2292.

19. Sun, Y.; Zhang, H.; Tao, W.; Ma, J.; Li, L.; Xia, J. Constant-Voltage Inductively Coupled Power Transfer System with Wide Load Range Based on Variable Structure Mode. Autom. Electr. Power Syst. 2016, 40, 109-114.

20. Qu, X.; Han, H.; Wong, S.C.; Tse, C.K.; Chen, W. Hybrid IPT topologies with constant current or constant voltage output for battery charging applications. IEEE Trans. Power Electron. 2015, 30, 6329-6337. [CrossRef]

21. Mai, R.; Chen, Y.; Zhang, Y.; Yang, N.; Cao, G.; He, Z. Optimization of the Passive Components for an S-LCC Topology-Based WPT System for Charging Massive Electric Bicycles. IEEE Trans. Ind. Electron. 2018, 65, 5497-5508. [CrossRef]

22. Ji, L.; Wang, L.; Liao, C.; Li, S. Design of Electric Vehicle Wireless Charging System with Automatic Charging Mode Alteration at Secondary Side. Autom. Electr. Power Syst. 2017, 23, 143-148.

23. Liu, G.; Bai, J.; Cui, Y.; Li, Z.; Yue, C. Double-Sided LCL Compensation Alteration Based on MCR-WPT Charging System. Trans. China Electrotech. Soc. 2019, 34, 1569-1579.

24. Qu, X.; Jing, Y.; Han, H.; Wang, S.; Tes, C.K. Higher Order Compensation for Inductive-Power-Transfer Converters with Constant-Voltage or Constant-Current Output Combating Transformer Parameter Constraints. IEEE Trans. Power Electron. 2017, 32, 394-405. [CrossRef]

25. Li, S.; Li, W.; Deng, J.; Nguyen, T.D.; Mi, C. A double-sided LCC compensation network and its tuning method for wireless power transfer. IEEE Trans. Veh. Technol. 2015, 64, 2261-2273. [CrossRef]

26. Kan, T.; Nguyen, T.D.; White, J.C.; Malhan, R.K.; Mi, C. A New Integration Method for an Electric Vehicle Wireless Charging System Using LCC Compensation Topology: Analysis and Design. IEEE Trans. Power Electron. 2017, 32, 1638-1650. [CrossRef]

(C) 2019 by the authors. Licensee MDPI, Basel, Switzerland. This article is an open access article distributed under the terms and conditions of the Creative Commons Attribution (CC BY) license (http://creativecommons.org/licenses/by/4.0/). 